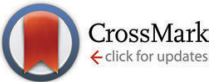

Cite this: Phys. Chem. Chem. Phys., 2015, 17, 25197

Received 22nd May 2015, Accepted 28th August 2015

DOI: $10.1039 / c 5 c p 02975 a$

www.rsc.org/pccp

\title{
A wavelength dependent investigation of the indole photophysics via ionization and fragmentation pump-probe spectroscopies
}

\author{
T. J. Godfrey, Hui Yu, Michael S. Biddle and Susanne Ullrich*
}

\begin{abstract}
A wavelength dependent study investigating the low-lying ${ }^{1} \mathrm{~L}_{a}$ and ${ }^{1} \mathrm{~L}_{b}$ states, both possessing ${ }^{1} \pi \pi^{*}$ character, and the ${ }^{1} \pi \sigma^{\star}$ state in the deactivation process of indole is presented here. Relaxation dynamics following excitation at 241, 250, 260, 270, 273, and $282 \mathrm{~nm}$ are examined using three gas-phase, pump-probe spectroscopic techniques: (1) hydrogen atom ( $\mathrm{H}$-atom) time-resolved kinetic energy release (TR-KER), (2) time-resolved photoelectron spectroscopy (TR-PES), and (3) time-resolved ion yield (TR-IY). Applied in combination, a more complete picture of the indole relaxation dynamics may be gleaned. For instance, TR-PES experiments directly observe all relaxation pathways by probing the evolution of the excited states following photoexcitation; whereas, TR-KER measurements indirectly, yet specifically, probe for ${ }^{1} \pi \sigma^{\star}$-state activity through the detection of $\mathrm{H}$-atoms eliminated along the indole nitrogen-hydrogen $(\mathrm{N}-\mathrm{H})$ stretch coordinate-a possible outcome of ${ }^{1} \pi \sigma^{\star}$-state relaxation in indole. In addition, mass information obtained via TR-IY monitors fragmentation dynamics that may occur within the neutral electronically excited and/or cationic states. The work herein assesses the onset and importance of the ${ }^{1} \pi \sigma^{\star}$ state at various pump wavelengths by systematically tuning across the ultraviolet absorption spectrum of indole with a particular focus on those pump wavelengths longer than $263 \mathrm{~nm}$, where the involvement of the ${ }^{1} \pi \sigma^{*}$ state is under current debate. As far as this experimental work is concerned, there does not appear to be any significant involvement by the ${ }^{1} \pi \sigma^{\star}$ state in the indole relaxation processes following excitation at 270,273 , or $282 \mathrm{~nm}$. This investigation also evaluates the primary orbital promotions contributing to the ${ }^{1} L_{a},{ }^{1} L_{b}$, and ${ }^{1} \pi \sigma^{*}$ transitions based on ionization preferences observed in TR-PES spectra. Relaxation time constants associated with dynamics along these states are also reported for excitation at all of the aforementioned pump wavelengths and are used to pinpoint the origin of the discrepancies found in the literature. In this context, advantages and disadvantages of the three experimental techniques are discussed.
\end{abstract}

\section{Introduction}

Procuring an exhaustive and, more importantly, consistent understanding of the aromatic heterocyclic molecule indole is immensely important as its presence in nature and application in biological processes is evident. For example, indole serves as a light-absorbing chromophore for the essential amino acid tryptophan and, as a building block of the eumelanin polymer, could play a vital role in preventing DNA photodamage linked to harmful ultraviolet (UV) radiation. Several investigations successfully demonstrate that gas-phase experiments may elucidate the photodynamics of certain molecules in the condensed phase $;^{3-7}$ hence, a series of gas-phase spectroscopic studies, which complement quantum chemical calculations, is a logical first step toward understanding indole's photodynamic properties in more complex environments and possibly its photoprotective role in eumelanin.

University of Georgia, Physics Building, Athens, Georgia, 30602, USA.

E-mail: ullrich@physast.uga.edu
An extensive amount of spectroscopic work exists, and the investigations by Townsend and co-workers, ${ }^{8}$ Stavros and Iqbal, ${ }^{9}$ Ashfold and co-workers, ${ }^{10}$ and Ullrich and co-workers ${ }^{11}$ provide excellent starting points for a more comprehensive review of the existing literature regarding the indole molecule. Here, we will focus primarily on those studies that investigate or aid in the understanding of the low-lying excited states with $\pi \pi^{*}$ and $\pi \sigma^{*}$ character that are accessible in our wavelength dependent study. The reported absorption spectra ${ }^{8,12-14}$ for the indole molecule are in very strong agreement. A key feature present in these studies is a broad peak spanning from approximately $220 \mathrm{~nm}$ to around $280 \mathrm{~nm}$. This signal is predominantly caused by absorption to a ${ }^{1} \pi \pi^{*}$ state, which is referred to as the ${ }^{1} \mathrm{~L}_{\mathrm{a}}$ state in accordance with Platt's nomenclature ${ }^{15}$ throughout the remainder of this report. Likewise, additional intensity extending to wavelengths longer than $280 \mathrm{~nm}$ is mainly due to absorption to the so-called ${ }^{1} \mathrm{~L}_{\mathrm{b}}$ state (also of ${ }^{1} \pi \pi^{*}$ character). The indole neutral ground state is denoted as $\mathrm{S}_{0}$ throughout the present study. 
In addition to the ${ }^{1} \mathrm{~L}_{\mathrm{a}}$ and ${ }^{1} \mathrm{~L}_{\mathrm{b}}$ states, other states may participate in the indole relaxation process as well. For example, if an electron from a $\pi$ (occupied bonding) orbital transitions to the $\sigma^{*}$ molecular orbital, an electronically excited $\pi \sigma^{*}$ state forms. As is the case in certain azoles, phenols, and larger heteroaromatic molecules, this type of excited state can aid in the nonradiative decay processes in indole. However, in contrast to some molecules like pyrrole or imidazole, where the $\pi \sigma^{*}$ state may be accessed directly, the $\pi \sigma^{*}$ state in indole is predominately populated indirectly via transfer from an optically bright state. $^{16}$

The transition to a $\sigma^{*}$ molecular orbital in indole can create a significant dipole moment of the $\pi \sigma^{*}$ state. For instance, Sobolewski and Domcke ${ }^{17}$ note in their discussion about indole that the charge distribution of the $\sigma^{*}$ molecular orbital is located beyond the atomic frame, and as such, the transition from the initial $\pi$ orbital to the $\sigma^{*}$ orbital possesses substantial charge-transfer characteristics, the result of which is a dipole moment of the ${ }^{1} \pi \sigma^{*}$ state. The authors also mention that this charge-transfer character coupled with the antibonding nodal character of the $\sigma^{*}$ orbital may lead to $\mathrm{H}$-atom dissociation from the nitrogen-hydrogen $(\mathrm{N}-\mathrm{H})$ moiety in indole-a process corroborated here and by several other experimental investigations. ${ }^{9-11,18}$

Sobolewski and Domcke also report potential energy profiles calculated using multiconfigurational second-order perturbation theory (CASPT2) ab initio techniques ${ }^{19}$ and at the CC2/aug-cc-pVDZ level. ${ }^{17}$ It should be noted that the results from both works are somewhat different. Their earlier study predicts an excitation energy of $4.30,4.65$, and $5.05 \mathrm{eV}$ for the ${ }^{1} \mathrm{~L}_{\mathrm{b}},{ }^{1} \mathrm{~L}_{\mathrm{a}}$, and ${ }^{1} \pi \sigma^{*}$ states, respectively. In contrast, the latter investigation reports a significant upward shift of the ${ }^{1} \mathrm{~L}_{\mathrm{b}}$-state and ${ }^{1} \mathrm{~L}_{\mathrm{a}}$-state minimums and a slight downward shift of the ${ }^{1} \pi \sigma^{*}$ minimum energy in comparison to their earlier work, creating a near degeneracy between all three states. Theoretical calculations by Townsend and co-workers ${ }^{8}$ are in strong agreement with Sobolewski and Domcke's latter work. ${ }^{17}$

An exhaustive amount of information exists regarding the ${ }^{1} \mathrm{~L}_{\mathrm{b}}$-state region, ${ }^{20-31}$ and numerous vibronic bands are identifiable between 4.37 , which is very close to the predicted ${ }^{1} \mathrm{~L}_{\mathrm{b}}$-state onset, and $\sim 4.58 \mathrm{eV}$. The experimentally determined ${ }^{1} \mathrm{~L}_{\mathrm{a}}$ onset is located at approximately $4.54 \mathrm{eV},{ }^{32,33}$ which is slightly lower than the predicted excitation energy of $4.65 \mathrm{eV}$ in Sobolewski and Domcke's earlier calculations and even lower than the value predicted by their latter work.

Indole relaxation dynamics may partially be explained by referencing the aforementioned ab initio calculations. According to the potential energy profiles calculated along the $\mathrm{N}-\mathrm{H}$ stretch coordinate, excitation to the ${ }^{1} \mathrm{~L}_{\mathrm{a}}$ state may indirectly populate the ${ }^{1} \pi \sigma^{*}$ state via an ${ }^{1} \mathrm{~L}_{\mathrm{a}}{ }^{1} \pi \sigma^{*}$ conical intersection (CI). Calculations and experiments performed by Schmitt and co-workers ${ }^{22,34}$ suggest an ${ }^{1} \mathrm{~L}_{\mathrm{a}} /{ }^{1} \mathrm{~L}_{\mathrm{b}} \mathrm{CI}$-accessible through Herzberg-Teller active modes and in very close vicinity to the ${ }^{1} \mathrm{~L}_{\mathrm{a}}$-state origin-provides an essentially "barrierless" mechanism for an ${ }^{1} \mathrm{~L}_{\mathrm{a}} \rightarrow{ }^{1} \mathrm{~L}_{\mathrm{b}}$ transition. The minimum-energy-path calculations of Giussani et al. ${ }^{35}$ substantiate these claims and clearly demonstrate the ${ }^{1} \mathrm{~L}_{\mathrm{b}} / \mathrm{S}_{0}$ CI that could mediate relaxation from the ${ }^{1} \mathrm{~L}_{\mathrm{b}}$ state back to the ground state. Thus, ${ }^{1} \mathrm{~L}_{\mathrm{a}} \rightarrow{ }^{1} \mathrm{~L}_{\mathrm{b}} \rightarrow \mathrm{S}_{0}$ and ${ }^{1} \mathrm{~L}_{\mathrm{a}} \rightarrow{ }^{1} \pi \sigma^{*} \rightarrow \mathrm{S}_{0}$ transitions are all possible relaxation mechanisms for the excited indole molecule, and, as mentioned earlier, relaxation along the ${ }^{1} \pi \sigma^{*}$ state may also lead to $\mathrm{H}$-atom dissociation from the $\mathrm{N}-\mathrm{H}$ bond.

In regard to the latter process, dissociation along the ${ }^{1} \pi \sigma^{*}$ relaxation pathway is not the only source of $\mathrm{H}$-atom photoproducts: Several works report $\mathrm{H}$-atom signal arising from statistical unimolecular decay from the vibrationally hot ground state. ${ }^{9-11,18}$ Because $\mathrm{H}$-atoms associated with ${ }^{1} \pi \sigma^{*}$-state activity are expected to carry higher kinetic energies than $\mathrm{H}$-atoms that emanate from statistical processes, signal associated with the ${ }^{1} \pi \sigma^{*}$ state may partially be isolated through kinetic energy analysis. This process is aptly demonstrated for indole in a previous work following excitation at $201 \mathrm{~nm} .{ }^{11}$

Several time-resolved experimental studies have been performed on the indole molecule to investigate the dynamics mentioned above, and Table 1 provides a summary of the more recent time constants associated with ${ }^{1} \mathrm{~L}_{\mathrm{a}}$-state (column 4 ), ${ }^{1} \mathrm{~L}_{\mathrm{b}}$-state (column 5), and ${ }^{1} \pi \sigma^{*}$-state (column 6 ) activity at various pump (column 2 ) and probe (column 3 ) wavelengths. The differing experimental techniques are also noted (column 1). It should be mentioned that Radloff and co-workers ${ }^{36}$ report no involvement of the ${ }^{1} \pi \sigma^{*}$ state in the indole relaxation process. Their photoelectron spectra and photoion results produce only long-lived components (greater than several hundred picoseconds), and the authors ascribe those times to the initially excited $\pi \pi^{*}$ state(s).

Table 1 helps accentuate the widespread disagreement among the reported values. Although disparate experimental techniques and various probing schemes are utilized, it is not unreasonable to believe that closer agreement among the values could be achieved-or at least a more visible trend if one exists. In reference to the aforementioned potential energy profile calculations, ${ }^{8,17,19,34,35}$ the extracted time constants are expected to be somewhat dependent on the pump wavelengths partially due to potential energy barriers that exist within the electronically excited states.

It is the aim of this work to investigate the excited-state relaxation dynamics of the indole molecule via a wavelength dependent study spanning a region above and below the ${ }^{1} \pi \sigma^{*}$ state onset. This work starts with a series of H-atom timeresolved kinetic energy release (TR-KER) experiments, where $\mathrm{H}$-atoms that photodissociate from the indole molecule are measured. As mentioned earlier, $\mathrm{H}$-atom signal associated with ${ }^{1} \pi \sigma^{*}$ activity in the electronically excited state is distinguishable from signal resulting from other processes on a kinetic energy basis. Thus an indirect analysis of ${ }^{1} \pi \sigma^{*}$ dynamics is achieved. More specifically, the point at which ${ }^{1} \pi \sigma^{*}$-state relaxation leads to $\mathrm{H}$-atom dissociation is identified. The associated appearance times of this signal at various excitation energies above this onset is also gleaned and the extracted time constants, as is demonstrated by an earlier work following excitation at $201 \mathrm{~nm},{ }^{11}$ may be viewed as a good estimate for the ${ }^{1} \pi \sigma^{*}$-state relaxation time. Our investigation then progresses to a complementary sequence of time-resolved photoelectron spectroscopy (TR-PES) experiments, which afford the unique capability of measuring directly all excited states involved in the indole deactivation process. Finally, a time-resolved ion yield (TR-IY) 
Table 1 The time constants presented here for the given pump $\left(\lambda_{\text {pu }}\right)$ and probe $\left(\lambda_{\text {pr }}\right)$ wavelengths are obtained using a variety of experimental techniques: time-resolved ion yield (TR-IY), time-resolved photoelectron spectroscopy (TR-PES), and, specific to H-atom photoproduct detection, timeresolved velocity map imaging (TR-VMI) and time-resolved kinetic energy release (TR-KER). Time constants obtained by our group are shown in bold

\begin{tabular}{|c|c|c|c|c|c|}
\hline Technique & $\lambda_{\mathrm{pu}}(\mathrm{nm})$ & $\lambda_{\mathrm{pr}}(\mathrm{nm})$ & ${ }^{1} \mathrm{~L}_{\mathrm{a}}$ & ${ }^{1} \mathrm{~L}_{\mathrm{b}}$ & ${ }^{1} \pi \sigma^{*}$ \\
\hline TR-PES & $\mathbf{2 0 1}^{b}$ & 294 & $<100$ fs & $23 \pm 5$ ps & $405 \pm 76 \mathrm{fs}$ \\
\hline TR-KER & $\mathbf{2 0 1}^{b}$ & 243.15 & $\mathbf{N} / \mathbf{A}$ & N/A & $367 \pm 39 \mathrm{fs}$ \\
\hline TR-IY & $235^{c}$ & 1305 & - & $150 \pm 20 \mathrm{ps}$ & - \\
\hline TR-IY & $239^{d}$ & 394 & - & $>100 \mathrm{ps}$ & - \\
\hline TR-PES & 241 & 294 & $<100$ fs & $371 \pm 85$ ps & $436 \pm 157 \mathrm{fs}$ \\
\hline TR-IY & $248^{c}$ & 1305 & $22 \pm 9 \mathrm{fs}$ & ps & $460 \pm 145 \mathrm{fs}$ \\
\hline TR-PES & $249^{e}$ & 300 & $<100$ fs & 350 ps $( \pm 20 \%)$ & 700 fs $( \pm 20 \%)$ \\
\hline TR-PES & 250 & 294 & $<100$ fs & $616 \pm 64 \mathrm{ps}$ & $413 \pm 144$ fs \\
\hline TR-PES & $250^{d}$ & 400 & - & $>100 \mathrm{ps}$ & - \\
\hline TR-IY & $250^{d}$ & 400 & - & $>100 \mathrm{ps}$ & - \\
\hline TR-KER & 250 & 243.15 & $\mathbf{N} / \mathbf{A}$ & $\mathbf{N} / \mathbf{A}$ & $671 \pm 84$ fs \\
\hline TR-IY & $255^{d}$ & 310 & - & $>100 \mathrm{ps}$ & - \\
\hline TR-PES & 270 & 294 & $272 \pm 83$ fs & ns & - \\
\hline TR-IY & $272.5^{c}$ & 1305 & $42 \pm 9$ fs & ns & - \\
\hline TR-PES & 273 & 294 & $276 \pm 142$ fs & ns & - \\
\hline TR-PES & $273^{e}$ & 300 & $<100 \mathrm{fs}$ & $\infty$ & $1200 \mathrm{fs}( \pm 20 \%)$ \\
\hline TR-IY & $278^{c}$ & 1305 & - & ns & - \\
\hline TR-PES & 282 & 294 & - & $\infty$ & - \\
\hline
\end{tabular}

${ }^{a}$ Data from ref. 9. ${ }^{b}$ Data from ref. 11. ${ }^{c}$ Data from ref. 59. ${ }^{d}$ Data from ref. 36, this work does not distinguish between ${ }^{1} \mathrm{~L}_{\mathrm{a}}$ - and ${ }^{1} \mathrm{~L}_{\mathrm{b}}$-state relaxation dynamics but instead associates the several hundred picoseconds time constant with relaxation from the initially prepared $\pi \pi^{*}$ state(s)—assuming simultaneous excitation to both states. ${ }^{e}$ Data from ref. 8.

experiment is also conducted to monitor fragmentation dynamics within the excited state and to confirm that the resulting photoelectron signal is, in fact, originating from indole, not an indole photoproduct. Through a conglomerate analysis involving these three techniques, we aim to clarify the energetic onset of ${ }^{1} \pi \sigma^{*}$-state involvement in the indole deactivation process and to decipher the timescales on which indole relaxation dynamics occur at all the aforementioned pump wavelengths. We also elaborate further on the suitability of different probe techniques for monitoring excited-state relaxation dynamics and propose an explanation for the inconsistent time constants found in the literature.

\section{Experimental details}

The experimental setup consists of a Ti : Sa oscillator (Coherent Inc. MIRA Model 900-F, which is pumped with a Coherent Inc. Verdi G5), and a regenerative amplifier (Coherent Inc. Legend Elite HE). Details regarding the setup are provided elsewhere, ${ }^{11}$ and deviations from previous investigations and essential experimental parameters are described as follows. All pump beams utilized in this investigation $(241,250,260,270,273$, and $282 \mathrm{~nm}$ ) are generated using a Coherent Inc. OPerA optical parametric amplifier and span above and below the region where the ${ }^{1} \pi \sigma^{*}$-state relaxation pathway becomes an energetically viable avenue for indole deactivation. The probe beams $(294 \mathrm{~nm}$ for TR-PES and TR-IY; $243.15 \mathrm{~nm}$ for TR-KER) are generated with a
Coherent Inc. TOPAS-C with UV extension. A probe of $294 \mathrm{~nm}$ is applied for TR-IY and TR-PES studies due to its extremely low absorbance in indole $e^{8,12-14}$-reducing the amount of undesired probe-pump signal. Pump and probe beams are generated with pulse energies around 10 and $30 \mu \mathrm{J}$ per pulse, respectively. Before intersecting the molecular beam, the pump pulse energy is reduced to a modest level $(<1.7 \mu \mathrm{J}$ per pulse) and the probe to approximately 7.0 $\mu \mathrm{J}$ per pulse for all TR-PES and TR-IY investigations. In regard to the TR-KER investigations, the probe beam pulse energy is set higher and centered around $243.15 \mathrm{~nm}$ to promote $\mathrm{H}$-atom detection, which relies on $2+1$ resonance enhanced multiphoton ionization (REMPI) via the $2 \mathrm{~s}$ state for neutral $\mathrm{H}$-atoms. Scanning the relative pump-probe delay times is achieved with a motorized delay stage (Newport ILS150PP with ESP300 controller), which is positioned in the TOPAS-C beam path.

A custom-built multipurpose spectrometer is used to record all measurements, and is described in detail elsewhere. ${ }^{37,38} \mathrm{~A}$ magnetic bottle spectrometer, based on the principles delineated by Kruit and Read, ${ }^{39}$ facilitates the detection of both photoelectrons and $\mathrm{H}$-atoms. $\mathrm{A}+14 \mathrm{~V}$ repelling potential is applied between extraction grids located above and below the ionization region (see ref. 11 for details) to improve $\mathrm{H}$-atom collection performance. The time-of-flight (TOF) mass spectrometer affords measurements of photoions and is based on the Wiley-McLaren configuration. ${ }^{40}$ TR-IY investigations use a $+1.5 \mathrm{kV}$ potential difference between the ion repeller and extraction grid and 
a $-1.5 \mathrm{kV}$ potential difference between the extraction and acceleration grids. A field-free region is generated within the flight tube by setting the liner to the acceleration grid potential. The indole sample ( $\geq 99 \%)$ is purchased directly from SigmaAldrich, Inc. and is used without further purification. The sample is heated to $35{ }^{\circ} \mathrm{C}$ in a differentially pumped vacuum chamber for all scans, and the resulting indole vapor is co-expanded and transported with a helium carrier gas to the ionization region of the spectrometer. A 50:50 mixture of 1,3-butadiene and helium is used to find the Gaussian cross-correlation in each experiment: the results of which are reported below in their respective sections (see Section A for TR-KER results and Section B for TR-PES in Results and Discussion). Photoelectron energy spectra are calibrated with the same butadiene-helium mixture and a $50: 50$ mixture of ammonia and helium using the photoelectron spectra in ref. 41 and 42 , respectively.

\section{Results and discussion}

A complementary TR-IY scan under otherwise identical experimental conditions was conducted for all of the investigations discussed in detail below. In each case, the resulting mass spectra confirmed the dominating presence of indole in the gas phase. With the chosen pump and probe powers, a negligible amount of fragmentation (i.e., anything other than $\mathrm{H}$-atom dissociation from the $\mathrm{N}-\mathrm{H}$ moiety) is observed in all cases and does not require consideration in our analyses. As an additional note, the parent ion signal in the TR-IY scans were well described with the same Gaussian convoluted exponential decay functions and time constants (within the given errors) as the TR-PES data presented below.

\section{A. Time-resolved kinetic energy release}

The steady-state H-atom kinetic energy spectra of indole photodissociated at 241, 250, 260, and $273 \mathrm{~nm}$ are discussed here.
In each case, the probe wavelength is centered at $243.15 \mathrm{~nm}$. The Gaussian cross-correlations, as measured within the spectrometer, are as follows: $248 \pm 7,223 \pm 9,218 \pm 9$, and $181 \pm 8$ fs, respectively. As mentioned in the Introduction, H-atoms deriving from direct dissociation along the ${ }^{1} \pi \sigma^{*}$ state are easily distinguished from $\mathrm{H}$-atoms produced via statistical unimolecular decay on $\mathrm{S}_{0}$ based on their kinetic energies. To demonstrate this, $\mathrm{H}$-atom kinetic energy spectra recorded at 273 and $250 \mathrm{~nm}$ are displayed in Fig. 1(a) and (b), respectively. Contributions from statistical decay are fitted with a Boltzmann distribution (blue) whereas direct dissociation products are described by a Gaussian (red). Clearly, a bimodal distribution is observed at $250 \mathrm{~nm}$ but the higher kinetic energy peak is noticeably absent at $273 \mathrm{~nm}$; indications of high-energy $\mathrm{H}$-atom signal become visible in the low- to mid-260 nm range but signal levels are insufficient for further analysis. All of the recorded spectra discussed here resemble low-resolution versions of those attained by Ashfold and co-workers ${ }^{10}$ and reproduce their reported onset for direct $\mathrm{H}$-atom elimination. In reference to our discussion in Section B, we briefly note that $\mathrm{H}$-atom detection experiments do not exclude relaxation to $S_{0}$ via the ${ }^{1} \pi \sigma^{*}$ state below this onset. In general, our $\mathrm{H}$-atom kinetic energy spectra support earlier high resolution work ${ }^{10}$ but do not contribute any additional spectroscopic information. Therefore, the following discussion focuses on the dynamics aspect of direct $\mathrm{N}-\mathrm{H}$ photodissociation at 241, 250, and $260 \mathrm{~nm}$. Integration over the high-energy $\mathrm{H}$-atom signal in our TR-KER spectra yields the delay traces depicted in Fig. 2, which are then fitted with a Gaussian convoluted singlestep function to extract photoproduct appearance times.

As shown in Fig. 2(a), a high-energy H-atom rise time of $\tau_{241}=515 \pm 63 \mathrm{fs}$ is obtained following excitation at $241 \mathrm{~nm}$. When the excitation wavelength increases to $250 \mathrm{~nm}$ (see Fig. 2(b)), the rise time associated with the high-energy H-atoms increases to $\tau_{250}=671 \pm 84$ fs. The trend continues when the wavelength is again raised to $260 \mathrm{~nm}$, producing a rise time of $\tau_{260}=932 \pm 91 \mathrm{fs}$ as presented in Fig. $2(\mathrm{c})$. While the measured
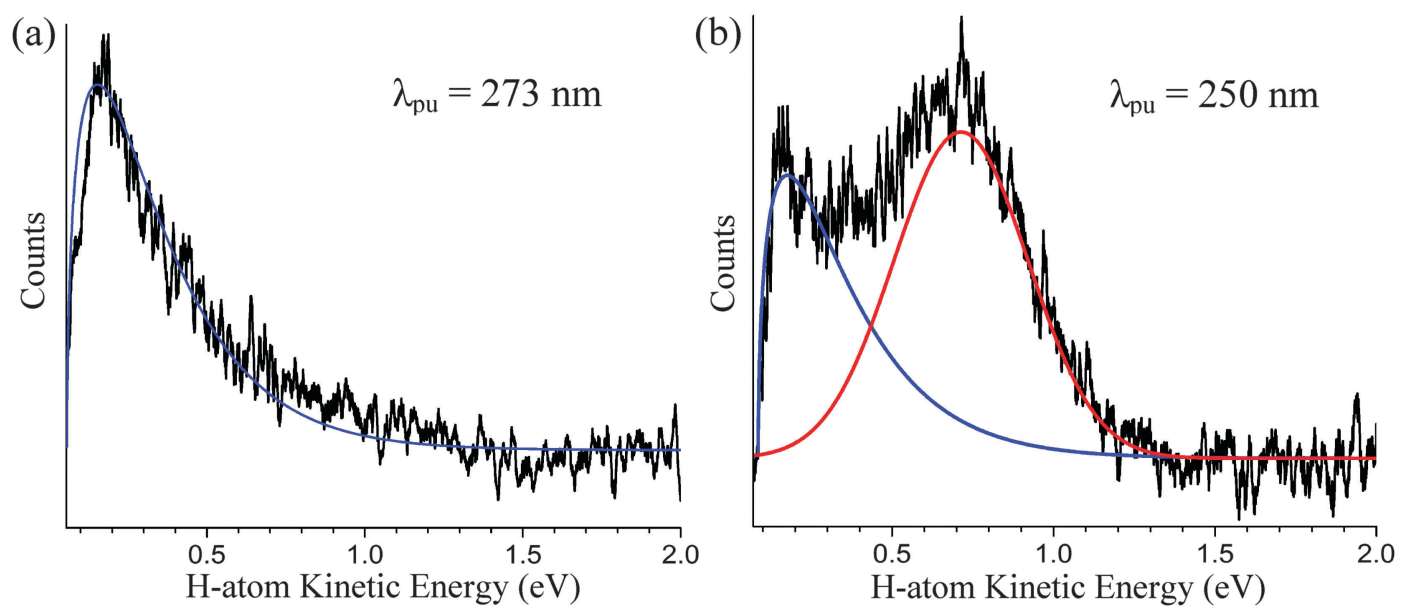

Fig. 1 The steady-state $\mathrm{H}$-atom kinetic energy spectra of indole photodissociated at (a) $273 \mathrm{~nm}$ and (b) $250 \mathrm{~nm}$ are presented here to highlight the presence of a high-energy $\mathrm{H}$-atom distribution at pump wavelengths shorter than $\sim 263 \mathrm{~nm}$. The low-energy signal in both figures is fitted with a statistical function (shown in blue) while the high-energy $\mathrm{H}$-atom distribution, associated with direct $\mathrm{N}-\mathrm{H}$ dissociation along the ${ }^{1} \pi \sigma^{*}$ state, is fitted with a Gaussian function (shown in red). 

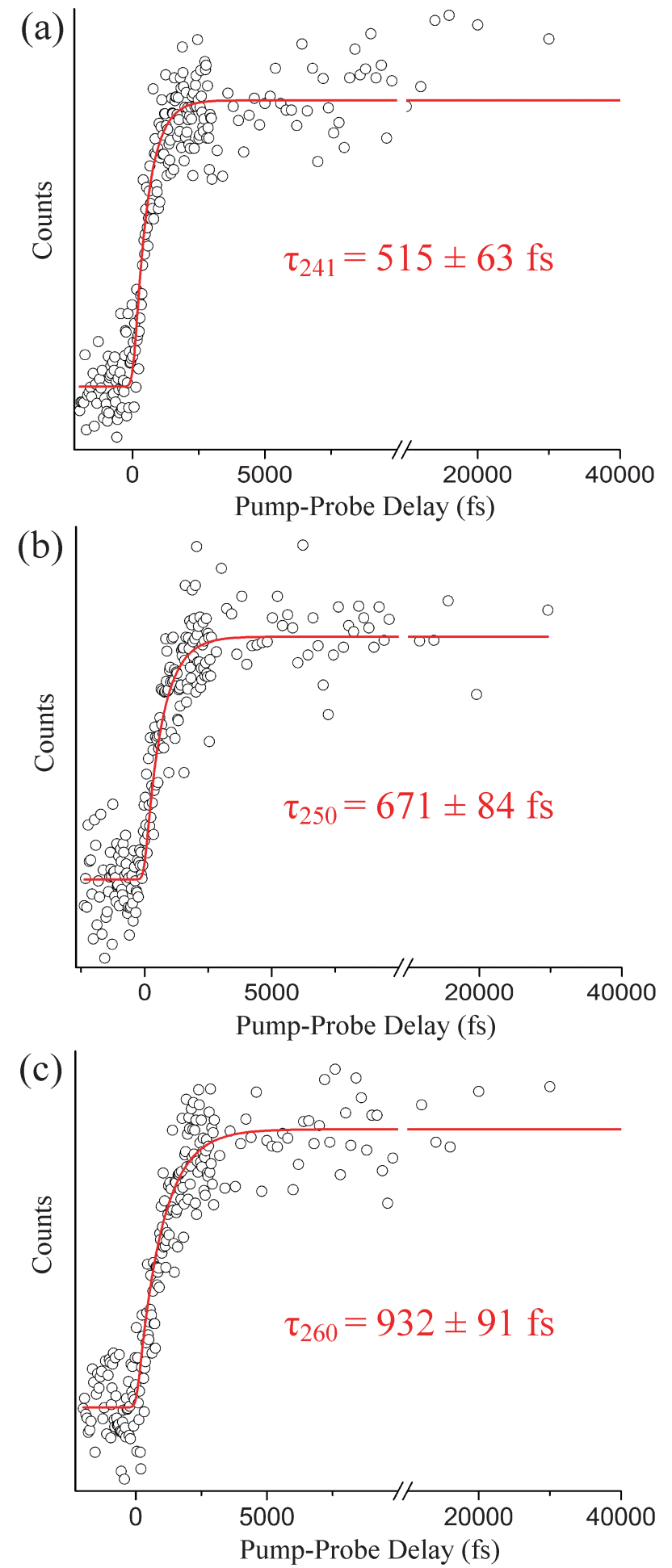

Fig. 2 Delay traces associated with the high-energy $\mathrm{H}$-atom distributions are shown here for pump wavelengths centered at (a) $241 \mathrm{~nm}$, (b) $250 \mathrm{~nm}$, and (c) $260 \mathrm{~nm}$. A clear increasing trend in photoproduct rise time is visible as the pump wavelength increases.

time constants correspond to the appearance of the $\mathrm{H}$-atom photoproduct, it may be regarded as an indirect measurement of relaxation along the ${ }^{1} \pi \sigma^{*}$ state. As such, the increase in rise time may be suggestive of a barrier existing along the ${ }^{1} \pi \sigma^{*}$ potential energy profile, as predicted by Townsend and co-workers ${ }^{8}$ and Sobolewski and Domcke, ${ }^{17,19}$ which is approached at the longer excitation wavelengths. Although both authors agree a barrier may exist, the height of the barrier is still unsure (ranging from $0.1 \mathrm{eV}$ to approximately $0.5 \mathrm{eV}$ ). This picture is revisited in the discussion below (Section B) when TR-KER and TR-PES results are compared and an alternative explanation is offered.

\section{B. Time-resolved photoelectron spectroscopy}

The photoelectron spectra presented here are taken at pump wavelengths of 241, 250, 260,270,273, and $282 \mathrm{~nm}$. A $294 \mathrm{~nm}$ probe is applied in all cases. A short-range scan with pumpprobe delays extending $\sim 4000$ fs beyond time-zero with a step size of $25 \mathrm{fs}$ is reported here at all pump wavelengths. A second set of experiments with pump-probe delays expanding beyond 100 ps with larger, unequal step sizes are also discussed at all pump wavelengths below $282 \mathrm{~nm}$. The Gaussian crosscorrelations for the short-range scans are $208 \pm 8,184 \pm 5$, $237 \pm 9,214 \pm 6,209 \pm 9$, and $172 \pm 8 \mathrm{fs}$, respectively. Likewise, the Gaussian cross-correlations for the long-range scans are $228 \pm 7,174 \pm 6,237 \pm 9,214 \pm 6$, and $201 \pm 5$ fs, respectively. Each short-range scan is deconvoluted into its individual contributions (channels) using a custom LabVIEW program based on a Levenberg-Marquardt optimization algorithm, which simultaneously fits spectra and associated decay dynamics along the energy and pump-probe delay axes. The number of channels assigned in each analysis is based on the necessity to accurately fit the data (i.e., the lowest number of channels where any residual signal is negligible and random noise). The results are then interpreted by considering ionization correlations (discussed in more detail shortly) and comparing our experimental outcomes to the numerous theoretical investigations. ${ }^{8,17,19,34,35,43-45}$ Three excited-state channels are needed to accurately fit the data attained at pump wavelengths centered around 241, 250, and $260 \mathrm{~nm}$. At 270 and $273 \mathrm{~nm}$, only two excited-state channels are required to accurately fit the results and only one at $282 \mathrm{~nm}$. It is assumed in all cases at or shorter than $273 \mathrm{~nm}$ that the ${ }^{1} \mathrm{~L}_{\mathrm{a}}$ state is excited almost exclusively, thus a rise time of zero femtoseconds is fixed for the ${ }^{1} \mathrm{~L}_{\mathrm{a}}$-state contribution throughout our analysis. To account for sequential dynamics from the ${ }^{1} \mathrm{~L}_{\mathrm{a}}$ state, rise times for the ${ }^{1} L_{b}$-state and ${ }^{1} \pi \sigma^{*}$-state contributions, when applicable, remain open during the fitting process and consistently converge to values similar to the ${ }^{1} \mathrm{~L}_{\mathrm{a}}$-state decay times. Pumping at $282 \mathrm{~nm}$ corresponds to direct excitation of the ${ }^{1} \mathrm{~L}_{\mathrm{b}}$ state (i.e., energetically below the ${ }^{1} \mathrm{~L}_{\mathrm{a}}$ onset), hence the rise time associated with the ${ }^{1} \mathrm{~L}_{\mathrm{b}}$ state is fixed at zero femtoseconds in this case.

Similarly, the long-range scans acquired at pump wavelengths of 241,250 , and $260 \mathrm{~nm}$ are accurately fitted with a Gaussian convoluted triple exponential decay function, and those at 270 and $273 \mathrm{~nm}$ with a Gaussian convoluted double exponential decay function. Again, exponential rises account for the sequential dynamics. The long-range scans are necessary to accurately extract time constants associated with the longlived ${ }^{1} \mathrm{~L}_{\mathrm{b}}$ channels.

In all cases, an additional channel with a negative decay constant is introduced to account for minor probe-pump 


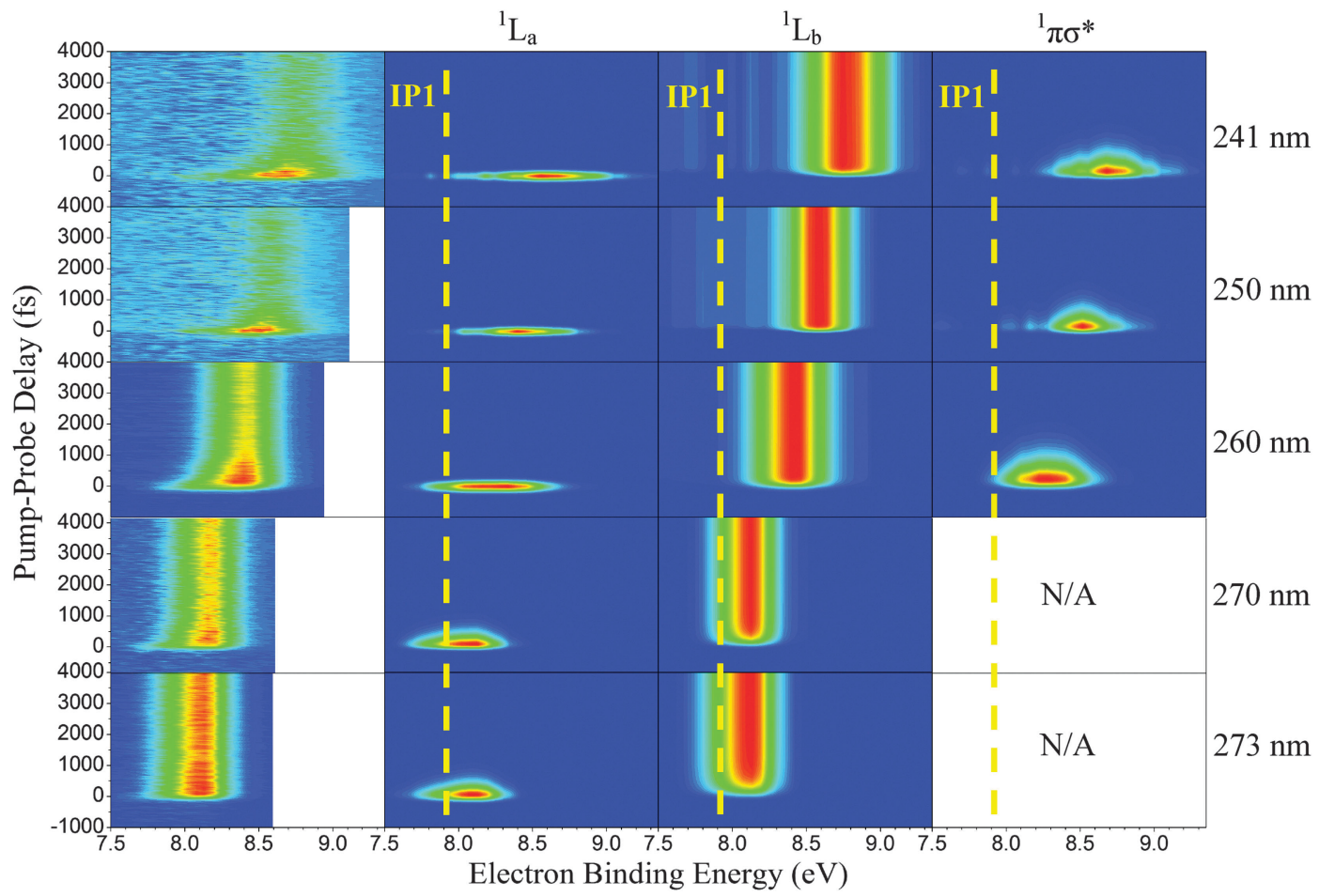

Fig. 3 Color maps are presented here for each ${ }^{1} \mathrm{~L}_{\mathrm{a}}$-state (column 2), ${ }^{1} \mathrm{~L}_{\mathrm{b}}$-state (column 3), and ${ }^{1} \pi \sigma^{*}$-state (column 4) contribution obtained through the global analysis of the raw TR-PES data (column 1) acquired at pump wavelengths of (from top to bottom) 241, 250, 260, 270, and 273 nm. The measured photoelectron kinetic energies are transformed to electron binding energies for comparison to the known indole ionization potentials. ${ }^{46-48}$ The yellowdashed lines mark the first indole vertical ionization potential at $7.90 \mathrm{eV}$.

signal; however, this channel is unrelated to the excited-state dynamics of interest and not discussed further.

The color maps depicted in Fig. 3 represent the extracted channels at pump wavelengths of $241,250,260,270$, and $273 \mathrm{~nm}$ as a function of pump-probe delay and electron binding energy. Due to differing dynamics and for clarification, the color maps corresponding to $282 \mathrm{~nm}$ excitation are shown separately in Fig. 4 . The electron binding energy is readily calculated by subtracting the measured photoelectron kinetic energies from the total pump and probe photon energies. In all cases, it is assumed only one photon from each beam is absorbed by the molecule, which can be justified for the laser powers and focusing conditions applied here. The first vertical ionization potential (labeled IP1) for indole is marked by the yellow-dashed line at $7.90 \mathrm{eV}^{46-48}$ in the second, third, and fourth columns of Fig. 3 and is used to help identify the electronically excited states. The first column in Fig. 3 displays the raw TR-PES signal for comparison to the individual contributions

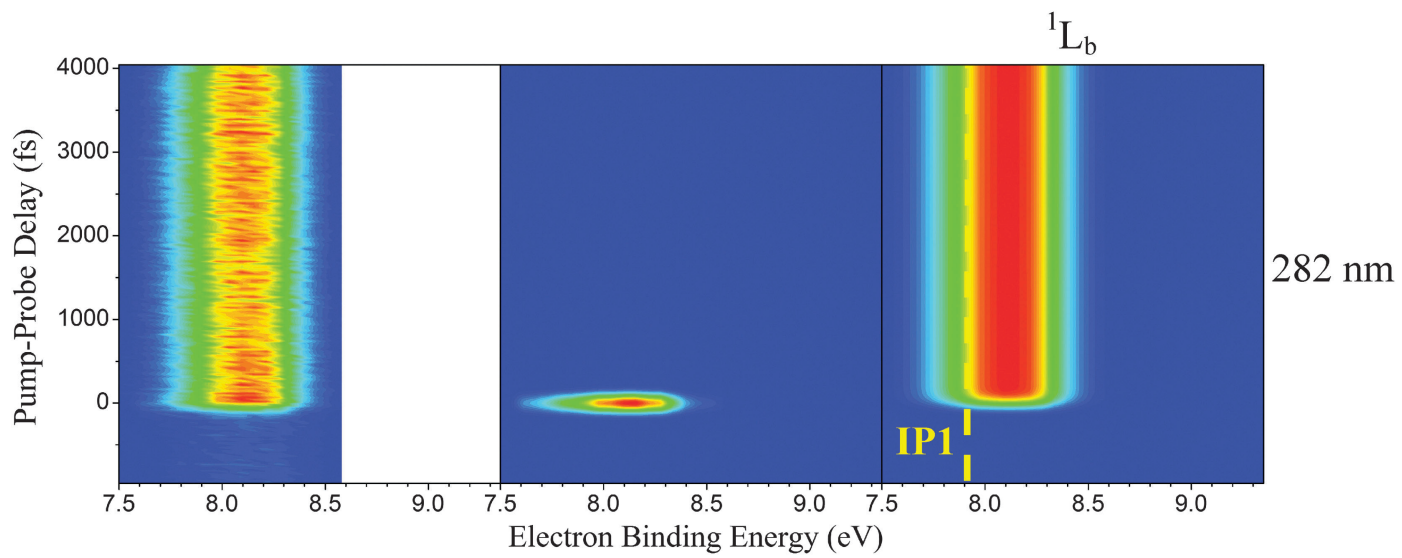

Fig. 4 Color maps of the raw TR-PES data set (column 1) and individual channels (columns 2 and 3) are shown for indole photoexcited at 282 nm. Measured photoelectron kinetic energies are transformed to electron binding energies for comparison to the known indole ionization potentials. ${ }^{46-48}$ The yellow-dashed line marks the first indole ionization potential at $7.90 \mathrm{eV}$. 
from each excited state. The second column consists of the channels associated with ${ }^{1} \mathrm{~L}_{\mathrm{a}}$-state deactivation to the ${ }^{1} \mathrm{~L}_{\mathrm{b}}$ state and, at shorter wavelengths, also the ${ }^{1} \pi \sigma^{*}$ state. As mentioned in an earlier investigation, ${ }^{11}$ the ${ }^{1} \mathrm{~L}_{\mathrm{a}} \rightarrow{ }^{1} \mathrm{~L}_{\mathrm{b}}$ and ${ }^{1} \mathrm{~L}_{\mathrm{a}} \rightarrow{ }^{1} \pi \sigma^{*}$ competing transitions occur simultaneously and are, in general, not discernable from one another due to spectral overlap in addition to similar ultrafast decay dynamics. Therefore, only a single time constant is assigned to the depopulation of the initially excited ${ }^{1} \mathrm{~L}_{\mathrm{a}}$ state. The third column in Fig. 3 presents a relatively long-lived signal associated with ${ }^{1} \mathrm{~L}_{\mathrm{b}}$-state relaxation to the $\mathrm{S}_{0}$ state. Column 4 encompasses the channels associated with deactivation along the ${ }^{1} \pi \sigma^{*}$ state, which is observed at pump wavelengths of 241, 250, and $260 \mathrm{~nm}$ but not at 270 or 273 .

Fig. 4 is structured similarly with the raw TR-PES signal presented in column 1 and contributing channels in columns 2 and 3. IP1 is again marked with a yellow-dashed line in column 3. As mentioned earlier, excitation at $282 \mathrm{~nm}$ directly excites the ${ }^{1} \mathrm{~L}_{\mathrm{b}}$ state; however, two channels are needed to accurately fit the data set: one accounting for ${ }^{1} \mathrm{~L}_{\mathrm{b}}$-state activity (excited-state channel) and another accounting for a Gaussianshaped contribution. We will elaborate on the assignments by examining the indole ionization correlations in the subsequent paragraphs.

The following discussion commences by examining indole ionization correlations. Because the ${ }^{1} \mathrm{~L}_{\mathrm{a}}$ state is populated from the highest occupied molecular orbital (HOMO) $)^{8,17,35,43,49}$ it is supposed that this state preferentially ionizes into the $\mathrm{D}_{0}$ state, which is associated with IP1 at $7.90 \mathrm{eV}$. In a very simple picture a $\Delta \nu=0$ propensity is assumed, where $\nu$ is a generalized quantum number and not mode specific, and any vibrational excitation in the ${ }^{1} \mathrm{~L}_{\mathrm{a}}$ state is transitioned to the ionic state. Therefore, shifts relative to the ionization potentials, in this case IP1, on an electron binding energy graph are expected. For example, the indole molecule is at first excited approximately $0.60 \mathrm{eV}$ above the ${ }^{1} \mathrm{~L}_{\mathrm{a}}$-state origin (located around $273 \mathrm{~nm}$ ) following absorption of a $241 \mathrm{~nm}$ photon. Assuming a $\Delta \nu=0$ propensity, we predict the maximum signal originating from the ${ }^{1} \mathrm{~L}_{\mathrm{a}}$ state to be positioned near $8.50 \mathrm{eV}$ (i.e., $0.60 \mathrm{eV}$ higher in binding energy than IP1), which is observed in our experimental work (see Fig. 3). Likewise, the ${ }^{1} \mathrm{~L}_{\mathrm{a}}$-state signal should exhibit shifts from approximately 0.42 to $0 \mathrm{eV}$ when pump wavelengths in the 250 to $273 \mathrm{~nm}$ region are employed. This expected and observed trend further ascertains our classification of these signal contributions as originating from the ${ }^{1} \mathrm{~L}_{\mathrm{a}}$ state and confirms an ${ }^{1} \mathrm{~L}_{\mathrm{a}} \rightarrow \mathrm{D}_{0}$ (IP1) ionization correlation.

As mentioned by Townsend et al. ${ }^{8}$ it may be assumed (based on several band decomposition studies ${ }^{50-54}$ ) that, almost exclusively, the ${ }^{1} \mathrm{~L}_{\mathrm{a}}$ state is excited in the pumping process, especially at the excitation wavelengths well above the ${ }^{1} \mathrm{~L}_{\mathrm{a}}$-state onset of $4.54 \mathrm{eV}$. As such, population of the ${ }^{1} \mathrm{~L}_{\mathrm{b}}$ state may solely be the result of relaxation from the ${ }^{1} \mathrm{~L}_{\mathrm{a}}$ state. Nevertheless, a similar analysis of the photoelectron spectra is implemented to study signals associated with the ${ }^{1} \mathrm{~L}_{\mathrm{b}}$ state. Based on the orbital calculations of Townsend et al., ${ }^{8}$ the ${ }^{1} \mathrm{~L}_{\mathrm{b}}$-state transition is expected to predominantly originate from the HOMO-1 and would therefore preferentially ionize into the $\mathrm{D}_{1}$ state, which should correspond with indole's second vertical ionization potential (IP2) of $8.35 \mathrm{eV}^{46-48}$ With the ${ }^{1} \mathrm{~L}_{\mathrm{b}}$-state origin taken to be at $283.8 \mathrm{~nm},{ }^{55}$ vibrational shifts in the approximate range of 0.77 to $0.03 \mathrm{eV}$ above IP2 would be expected as the pump wavelength increases from $241 \mathrm{~nm}$ to $282 \mathrm{~nm}$. As shown in Fig. 3 and 4, a general "shifting", trend is observed; however, the maximum signal peaks at all excitation wavelengths are consistently located below these predictions. Most notably for excitation at long wavelengths, the ${ }^{1} \mathrm{~L}_{\mathrm{b}}$ photoelectron signals fall below IP2. Townsend et al. observed a similar discrepancy in their analysis-an outcome they relate to the transmission efficiency of their spectrometer. Instead we challenge the proposed ${ }^{1} \mathrm{~L}_{\mathrm{b}} \rightarrow \mathrm{D}_{1}$ (IP2) ionization correlation. According to Platt's notation, ${ }^{15}$ the ${ }^{1} \mathrm{~L}_{\mathrm{b}}$ transition originates from LUMO $+1 \leftarrow$ HOMO and LUMO $\leftarrow$ HOMO- 1 excitations. Close inspection of the orbital representations associated with the HOMO and HOMO-1 reveals stark differences throughout the literature and coefficients describing the relative contributions of orbital promotions are also inconsistent. ${ }^{8,17,35,43,49,56}$ Given that theoretical descriptions are presently inconclusive, we propose an ${ }^{1} \mathrm{~L}_{\mathrm{b}} \rightarrow \mathrm{D}_{0}$ (IP1) ionization correlation based on our experimental observations. For instance, assuming ionization into the $\mathrm{D}_{0}$ (IP1) state, vibrational shifts in the approximate range of 0.77 to $0.03 \mathrm{eV}$ above IP1 would be expected as the pump wavelength increases from 241 to $282 \mathrm{~nm}$. This is observed in all cases (see Fig. 3 and 4). Furthermore, the $282 \mathrm{~nm}$ TR-PES spectrum in Fig. 4 clearly demonstrates ionization of ${ }^{1} \mathrm{~L}_{\mathrm{b}}$ into $\mathrm{D}_{0}$ (IP1) and further substantiates our proposal. A similar disparity exists for the ${ }^{1} \pi \sigma^{*}$-state ionization correlations and, following the same arguments, we also propose preferential ionization into $\mathrm{D}_{0}$ (IP1). In any case, assignment of the ${ }^{1} \pi \sigma^{*}$ channel is unambiguously supported by the above TR-KER measurements.

As shown in Fig. 4, two pump-probe channels are needed to accurately fit the raw data set acquired at $282 \mathrm{~nm}$. While the photoelectron spectra of both channels (columns 2 and 3) are very similar (i.e., they fall within the same electron binding energy range), a single exponential decay function would be insufficient to adequately describe the dynamics. With the excitation onset of the ${ }^{1} \mathrm{~L}_{\mathrm{a}}$ state located at $273 \mathrm{~nm}$, it is assumed at $282 \mathrm{~nm}$ that we are pumping directly into the ${ }^{1} \mathrm{~L}_{\mathrm{b}}$ state, and we associate the long-lived channel (column 3) with ${ }^{1} \mathrm{~L}_{\mathrm{b}}$-state activity because this contribution is highly consistent with the ${ }^{1} \mathrm{~L}_{\mathrm{b}}$-state channels of Fig. 3 and is congruous with our proposed ${ }^{1} \mathrm{~L}_{\mathrm{b}} \rightarrow \mathrm{D}_{0}$ (IP1) ionization correlation. Because $282 \mathrm{~nm}$ is slightly above the ${ }^{1} \mathrm{~L}_{\mathrm{b}}$-state onset, it is possible that the signal in column 2 corresponds to motion from the Franck-Condon region toward the ${ }^{1} \mathrm{~L}_{\mathrm{b}}$ minimum. Another likely cause is nonresonant ionization or out-of-resonance adiabatic excitation, which are not related to the excited-state dynamics of interest. A similar effect as these latter contributions has been observed by Longarte et al. in naphthalene and the related tryptophan molecule. ${ }^{57,58}$

We now focus our discussion on the associated time traces. The raw data sets, overall fits, and separate channel contributions associated with the short-range experiments administered at 241, 250, 260, 270, and $273 \mathrm{~nm}$ are displayed in Fig. 5. Results at $282 \mathrm{~nm}$ are shown in Fig. 6. We designate the green delay traces to 
relaxation originating from the ${ }^{1} \mathrm{~L}_{\mathrm{a}}$ state. All purple and black delay traces are attributed to relaxation along the ${ }^{1} \mathrm{~L}_{\mathrm{b}}$ state and ${ }^{1} \pi \sigma^{*}$ state, respectively. The black-dashed lines represent the aforementioned probe-pump contributions. In Fig. 6, the blue-dashed line depicts the Gaussian-shaped contribution.

Our discussion will begin with the results obtained following excitation at $241 \mathrm{~nm}$. Three time constants related to excited-state dynamics are needed to properly fit the obtained data set. The green delay trace furnishes the leading contribution to the overall signal and carries a relaxation time on an ultrafast timescale $\left(\tau_{1}<100 \mathrm{fs}\right)$. A second time constant, $\tau_{2}=436 \pm$ $157 \mathrm{fs}$, is also extracted from the fitting process and is denoted by the black delay trace. This lifetime, although still extremely fast, is notably longer than the first time constant. The third and final time constant, $\tau_{3}=371 \pm 85 \mathrm{ps}$, is from the longlived contribution represented by the purple delay trace. These results are in strong agreement with the TR-IY studies conducted by Longarte et al. ${ }^{59}$ at a pump wavelength of $243 \mathrm{~nm}$.

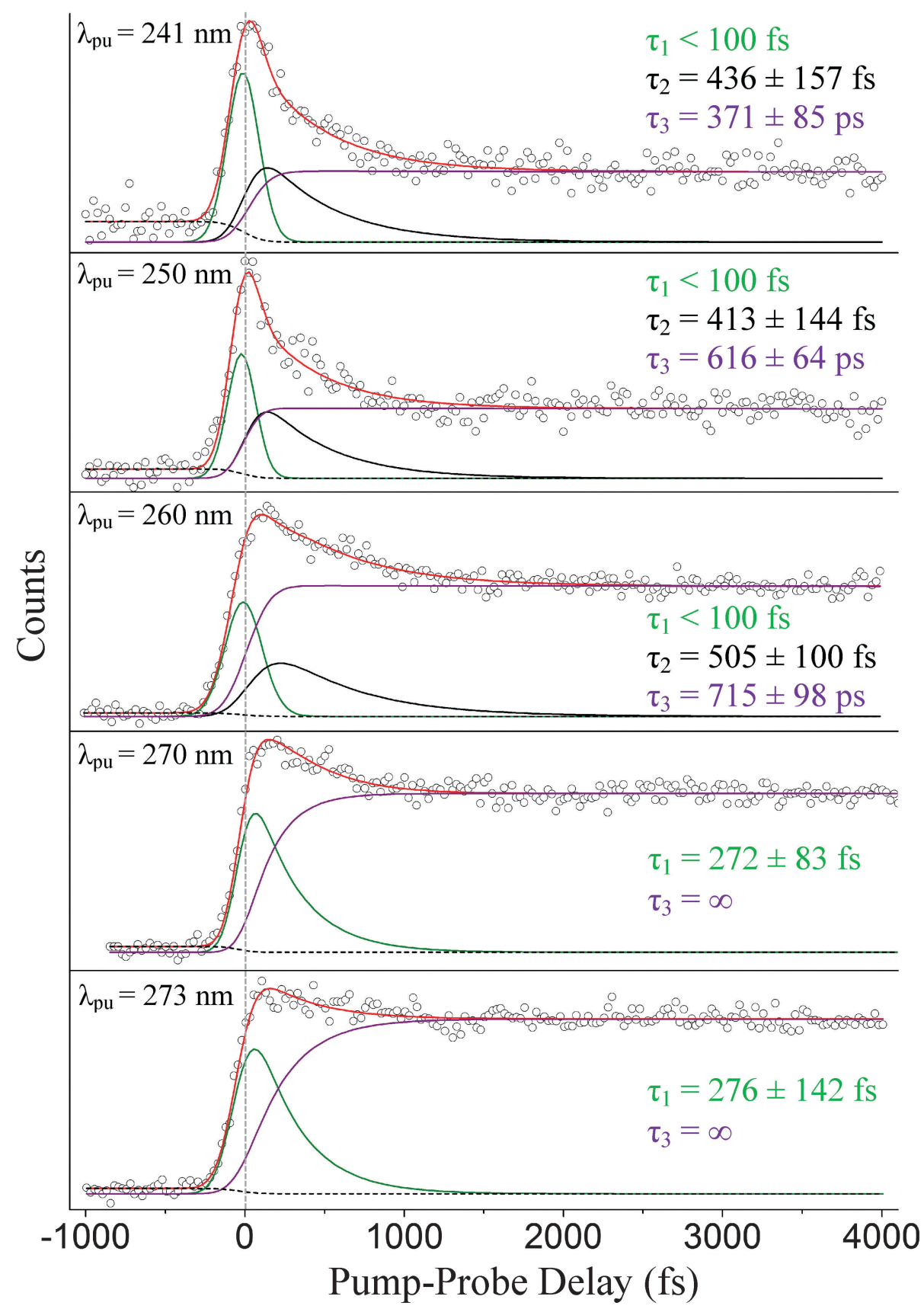

Fig. 5 Short-range TR-PES signal $(O)$, fits (red), and individual contributions $\left({ }^{1} L_{a}-\right.$ green, ${ }^{1} \pi \sigma^{*}-$ black, ${ }^{1} L_{b}-$ purple, and probe-pump signal - blackdashed) integrated over all photoelectron kinetic energies are shown at various pump wavelengths: (from top to bottom) 241, 250, 260, 270, and 273 nm. Relaxation times associated with each channel $\left({ }^{1} L_{a}-\tau_{1},{ }^{1} \pi \sigma^{*}-\tau_{2},{ }^{1} L_{b}-\tau_{3}\right)$ are also displayed in their respective graphs. The zero position is marked by the gray-dashed vertical line. 


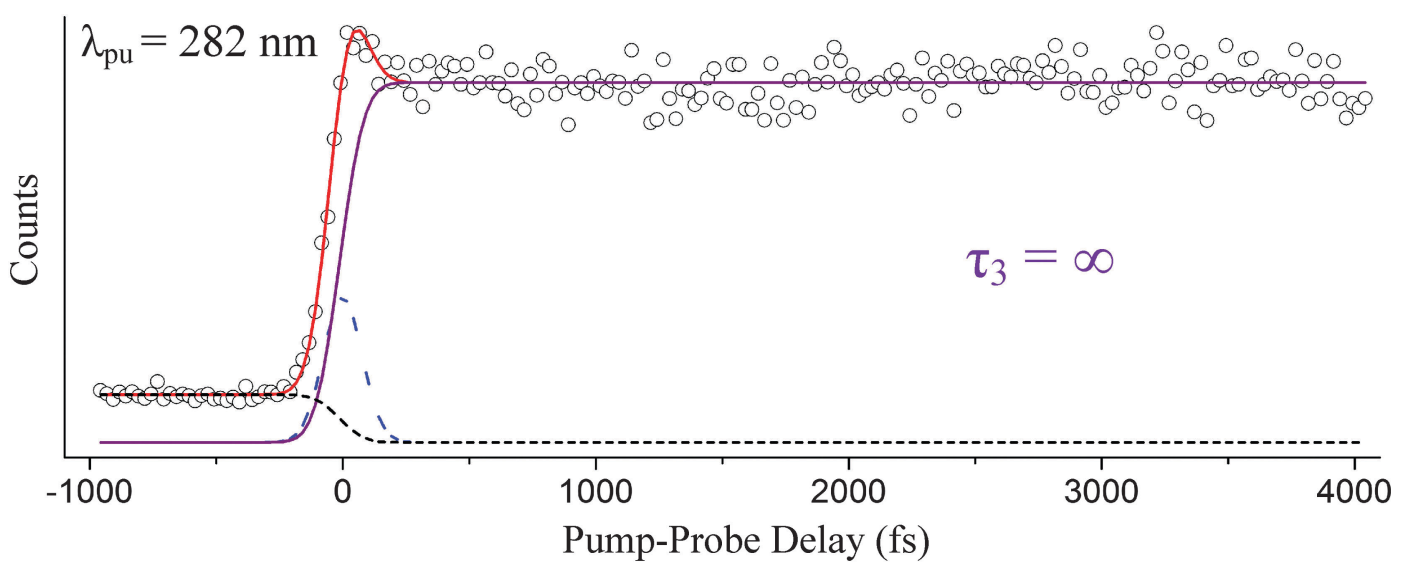

Fig. 6 Short-range TR-PES signal $(O)$, fit (red), and individual contributions $\left({ }^{1} L_{b}\right.$ - purple, nonresonant activity - blue-dashed, and probe-pump signal black-dashed) integrated over all photoelectron kinetic energies are shown following photoexcitation at $282 \mathrm{~nm}$.

Longarte et al. report time constants of $22 \pm 9$ fs (similar to our $\left.\tau_{1}\right), 435 \pm 125$ fs (in agreement with our $\tau_{2}$ ), and $315 \pm 50 \mathrm{ps}$ (in agreement with our $\tau_{3}$ ). It should be noted, however, that Longarte et al. employ a $1305 \mathrm{~nm}$ multiphoton probe, which, in principle, could produce contrasting dynamics if intermediate resonances are hit during the ionization process.

Because we associate $\tau_{2}$ with relaxation along the ${ }^{1} \pi \sigma^{*}$ state, this time constant can be compared to our independent $\mathrm{H}$-atom TR-KER results discussed in Section A. The time constants are in agreement within the reported error, although the TR-KER value is somewhat longer. While it is certainly possible that this difference corresponds to the additional time required for the formation of the fully separated photoproduct, another possible explanation is discussed below. Likewise, the separate longrange TR-PES scan from which $\tau_{3}$ derives is described very well with these same time constants (see Fig. 7).

At a pump wavelength centered around $250 \mathrm{~nm}$, three time constants associated with excited-state relaxation dynamics are gleaned: $\tau_{1}<100 \mathrm{fs}, \tau_{2}=413 \pm 144 \mathrm{fs}$, and $\tau_{3}=616 \pm 64 \mathrm{ps}$. Increasing the pump wavelength from $241 \mathrm{~nm}$ to $250 \mathrm{~nm}$ did not reveal any pronounced changes in $\tau_{1}$ or the lifetime associated with ${ }^{1} \pi \sigma^{*}$-state activity $\left(\tau_{2}\right)$; however, a significant increase in decay time is detected for the ${ }^{1} \mathrm{~L}_{\mathrm{b}}$-state $\left(\tau_{3}\right)$ signal, which is nearly double that at $241 \mathrm{~nm}$. Our result for $\tau_{1}$ is consistent with that obtained by Townsend et al., ${ }^{8}$ who report a value of $<100 \mathrm{fs}$ at a pump wavelength of $249 \mathrm{~nm}$, and Longarte et al., who report $22 \pm 9 \mathrm{fs}$ at a pump wavelength of $248 \mathrm{~nm}$. However, a noticeable difference between our result for $\tau_{2}$ and Townsend et al. is now made manifest. At essentially the same wavelengths as those applied here, Townsend et al. establish a lifetime of approximately $700 \mathrm{fs}$ for the ${ }^{1} \pi \sigma^{*}$ state, which is much different than our $\tau_{2}=413 \pm 144 \mathrm{fs}$. Our results are more comparable to those reported by Longarte et al., who report a ${ }^{1} \pi \sigma^{*}$-state lifetime of $460 \pm 145 \mathrm{fs}$. Unlike the case at $241 \mathrm{~nm}, \tau_{2}$ extracted via TR-PES does not corroborate the rise time $\tau_{250}=$ $671 \pm 84$ fs from the previously discussed TR-KER experiments in Section A. We believe the reason for this difference can be linked to the loss of the ionization window between the downward curving ${ }^{1} \pi \sigma^{*}$-state potential energy profile ${ }^{8,17,19}$ and the upward curving ionic-state potential as the $\mathrm{N}-\mathrm{H}$ bond length increases, ${ }^{60}$ an issue that has previously been raised in the literature for the case of adenine. ${ }^{61}$ Indirect measurements such as TR-KER are unaffected by this issue since the probe process relies on the photoionization of $\mathrm{H}$-atoms. It is therefore our conclusion that the TR-KER results presented in Section A are more representative of the actual relaxation times associated with ${ }^{1} \pi \sigma^{*}$ decay. Once the ${ }^{1} \pi \sigma^{*} / \mathrm{S}_{0} \mathrm{CI}$ has been passed, dissociation occurs quickly on the repulsive potential energy curve. Furthermore, the large discrepancy between TR-KER and TR-PES time constants suggests that a significant portion of the dynamics occur at extended $\mathrm{N}-\mathrm{H}$ distances, most likely in the vicinity of the $\mathrm{CI}$, where they are inaccessible to direct photoionization at the utilized probe wavelength.

It should also be noted that there is a substantial disagreement between our $\tau_{3}$ and the long-lived component measured by Townsend et $\mathrm{al}^{8}{ }^{8}$ These authors report a long-lived component with an associated lifetime of 350 ps, nearly half our value, following excitation at $249 \mathrm{~nm}$. One possible reason for this disparity is the maximum scanning range beyond time-zero. In principle, the extracted time constants should be independent of the selected scanning range, but it is our experience that values can vary significantly due to the presence of noise in real data sets. Townsend et al.'s scan range extends to approximately $50 \mathrm{ps}$; whereas, this investigation scans slightly over double that range to extract the long-lived contribution to the signal (see Fig. 7). The work of Longarte et $a .^{59}$ reports a value in the picosecond range; however, it is unclear where exactly in the picosecond range their value is located. It should also be noted that none of the studies observe the long-lived signal levels decaying back to a zero baseline. Further insight regarding this disparity may be gleaned by continuing this discussion following the reports at the remaining excitation wavelengths. As such, we will return to this comparison shortly.

Following excitation at $260 \mathrm{~nm}$, time constants of $\tau_{1}<100 \mathrm{fs}$, $\tau_{2}=505 \pm 100 \mathrm{fs}$, and $\tau_{3}=715 \pm 98 \mathrm{ps}$ are acquired. Again, no considerable change in $\tau_{1}$ or $\tau_{2}$ is observed in this case, but in comparison to Section A the difference between the TR-KER and TR-PES ${ }^{1} \pi \sigma^{*}$ time constants increases further. Longarte et al. ${ }^{59}$ report time constants of $22 \pm 9$ fs and $370 \pm 110$ fs for activity 


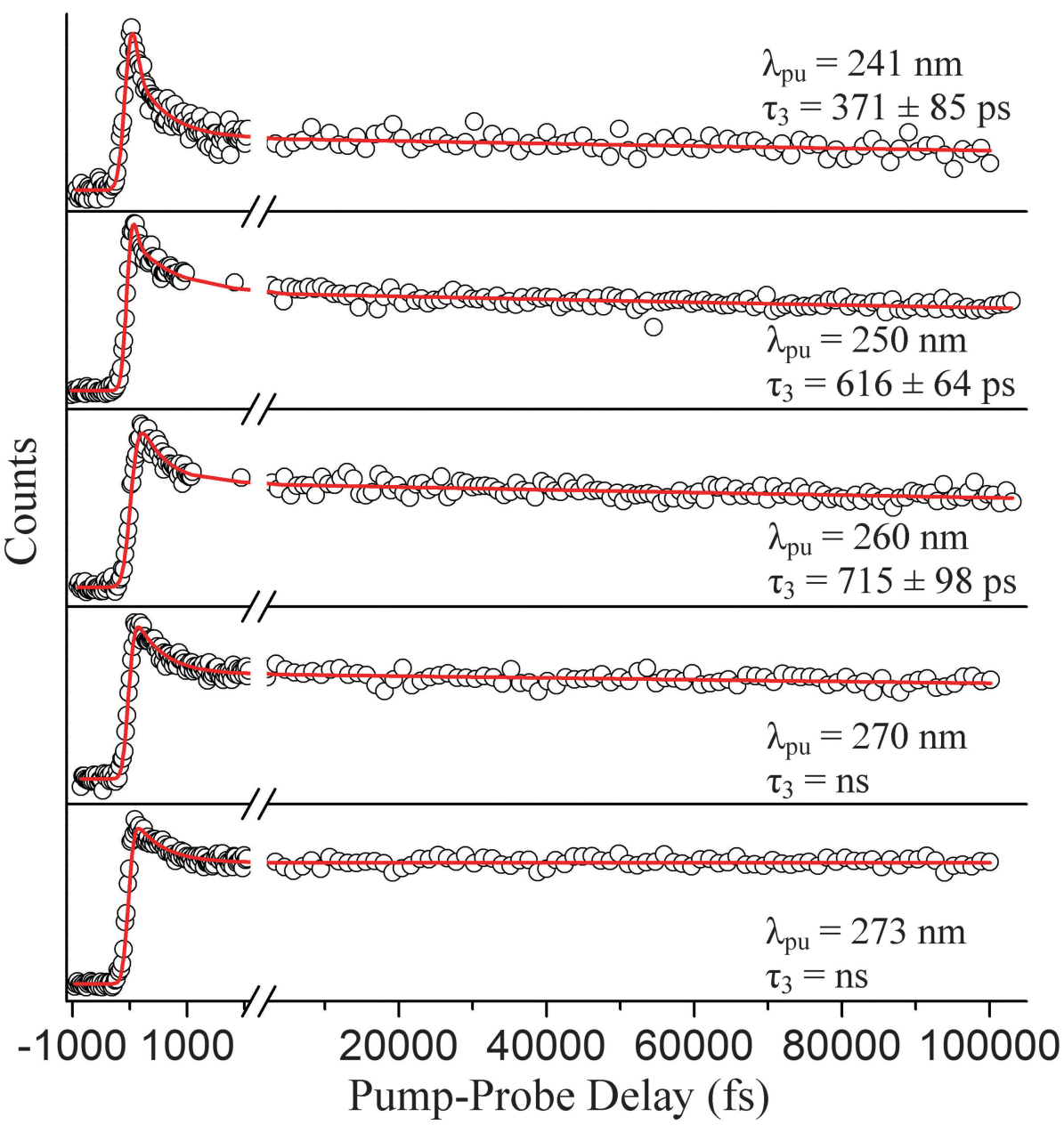

Fig. 7 TR-PES signal $(O)$ for the long-range scans and fits (red) integrated over all photoelectron kinetic energies are shown to complement the shortrange scans displayed in Fig. 5. The pump wavelengths here (from top to bottom) are 241, 250, 260, 270, and $273 \mathrm{~nm}$. Relaxation times associated with the ${ }^{1} L_{b} \rightarrow S_{0}$ transition $\left(\tau_{3}\right)$ are also displayed in their respective graphs.

correlated with ${ }^{1} \mathrm{~L}_{\mathrm{a}}$-state and ${ }^{1} \pi \sigma^{*}$-state deactivation, respectively, following excitation at the same pump wavelength. However, another dissimilarity presents itself between our $\tau_{3}=715 \pm 98$ ps and the work of Longarte et al. Scanning nearly 140 ps beyond time-zero, their work reports a lifetime of $7 \pm 2 \mathrm{~ns}$ associated with ${ }^{1} \mathrm{~L}_{\mathrm{b}}$-state relaxation. To address this discrepancy, a separate scan (not presented here) extending beyond 200 ps was conducted, and the extracted decay constants verify the values reported here. The reason for this difference is not clear but, as mentioned earlier, could be related to the scanning range or the multiphoton nature of the ionization process employed by Longarte et al. Further picosecond or nanosecond time-resolved studies may aspire to investigate the time constants associated with ${ }^{1} \mathrm{~L}_{\mathrm{b}}$-state relaxation following excitation at $260 \mathrm{~nm}$ to verify these claims.

Following excitation at $270 \mathrm{~nm}$, only two time constants are needed to accurately fit the data set: $\tau_{1}=272 \pm 83$ fs and $\tau_{3}=$ ns. Likewise at $273 \mathrm{~nm}$, two time constants associated with excited-state dynamics are extracted: $\tau_{1}=276 \pm 142$ fs and $\tau_{3}=$ ns. To our knowledge, no prior work associates similar time constants to ${ }^{1} \mathrm{~L}_{\mathrm{a}}$-state relaxation for excitation close to the origin. However, the aforementioned discussion regarding ionization correlations strongly supports this assignment. Similar to our previous scans, the separate long-range scan is also accurately fitted with these short-range time constants (see Fig. 7). These results challenge the assignment of an ultrafast time constant (i.e., $<100 \mathrm{fs}$ ) to ${ }^{1} \mathrm{~L}_{\mathrm{a}}$-state relaxation by Townsend et al. and Longarte et al. We refer to the potential energy profiles calculated along ring deformation coordinates by Giussani et $a l^{35}$ for further discussion. Their calculation positions the ${ }^{1} \mathrm{~L}_{\mathrm{a}} /{ }^{1} \mathrm{~L}_{\mathrm{b}}$ CI in very close vicinity to the ${ }^{1} \mathrm{~L}_{\mathrm{a}}$-state minimum which, at first sight, seems to strongly support an ultrafast transition. However, Giussani et al. report theoretical ${ }^{1} \mathrm{~L}_{\mathrm{a}}$ - and ${ }^{1} \mathrm{~L}_{\mathrm{b}}$-state minima that are lower than the experimental onsets according to ref. 32, 33 and 55 by approximately 0.14 and $0.26 \mathrm{eV}$, respectively. If these same curves are shifted upward to the known experimental values, the separation between the CI and the ${ }^{1} \mathrm{~L}_{\mathrm{a}}$-state minimum increases and our slightly longer $\tau_{1}$ constants are certainly not unreasonable.

As far as this experimental work is concerned, there is no apparent contribution originating from the ${ }^{1} \pi \sigma^{*}$ state at 270 and $273 \mathrm{~nm}$. The fact that a third excited-state relaxation channel is not necessary to accurately fit the short-range scans presented in Fig. 5 or the long-range scans in Fig. 7 supports 


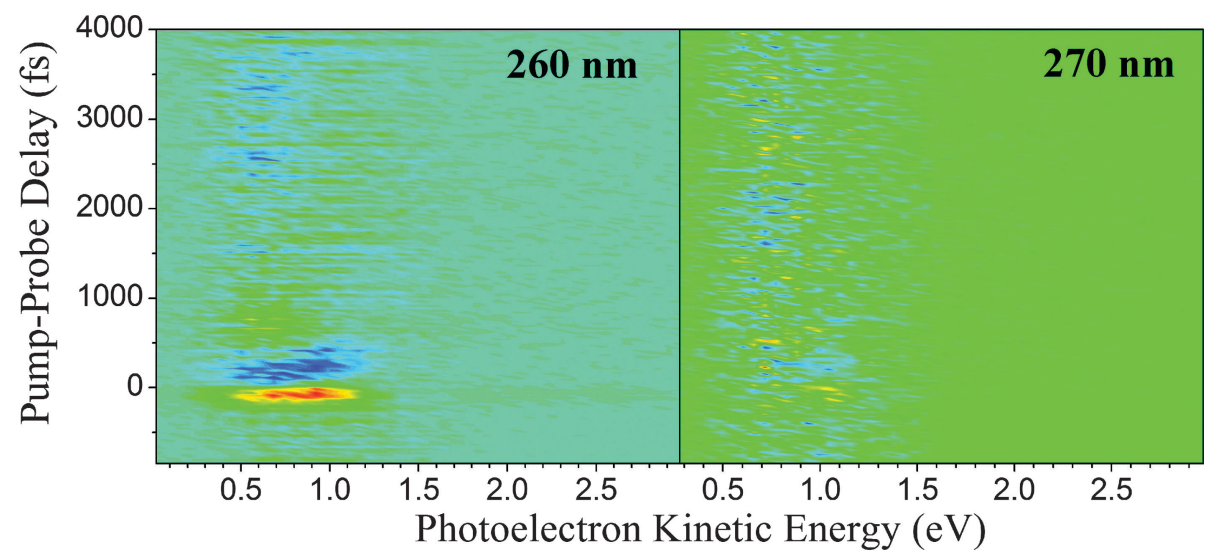

Fig. 8 When fitted with only two excited-state channels, the residual signal following excitation at $260 \mathrm{~nm}$ (left) clearly displays unaccounted for signal, thus requiring the inclusion of a third pump-probe channel. However, the photoelectron signal acquired at $270 \mathrm{~nm}$ is adequately described using only two pump-probe channels (right).

this claim. As mentioned earlier, the number of channels chosen at each pump wavelength is based on the necessity to obtain an accurate fit. For instance, when the data acquired at 241,250 , and $260 \mathrm{~nm}$ is fitted with only two excited-state channels, the residual signal clearly displays unaccounted for contributions to the overall signal, often in the ultrafast region of the spectrum. This is not the case at 270 and $273 \mathrm{~nm}$ where both data sets are accurately described using only two excitedstate channels. To visualize these effects, Fig. 8 selectively displays the residuals for the 260 and $270 \mathrm{~nm}$ TR-PES spectra when fit with only two pump-probe channels. We also note, that forcing an additional, unnecessary channel into the 270 and $273 \mathrm{~nm}$ fits leads to a spectral deconvolution into features that are incompatible with our general analysis (i.e., ionization correlations and vibrational shifts are inexplicable).

The ultrafast electron diffraction results reported by Zewail and co-workers ${ }^{62}$ bolster this interpretation as well. In their experimental work, the authors conclude that the ${ }^{1} \pi \sigma^{*}$ state does not play a significant role in indole relaxation dynamics following excitation at $267 \mathrm{~nm}$. This is in direct contrast with Townsend et al., ${ }^{8}$ who compare their results taken at $249 \mathrm{~nm}$, where the ${ }^{1} \pi \sigma^{*}$ state is actively involved in the relaxation process, and $273 \mathrm{~nm}$ to arrive at the conclusion that the ${ }^{1} \pi \sigma^{*}$ state is participating in the relaxation process at $273 \mathrm{~nm}$. The previously discussed TR-KER results (Section A) provide no evidence for ${ }^{1} \pi \sigma^{*}$-state activity at $273 \mathrm{~nm}$ either; however, the TR-KER measurements only identify the onset of $\mathrm{H}$-atom dissociation from the $\mathrm{N}-\mathrm{H}$ moiety along the ${ }^{1} \pi \sigma^{*}$ state, which may differ from the onset of ${ }^{1} \pi \sigma^{*} \rightarrow S_{0}$ relaxation. We do acknowledge that, in principle, it is possible for the ${ }^{1} \pi \sigma^{*}$ state to actively participate in the relaxation process at these longer wavelengths without breaking the $\mathrm{N}-\mathrm{H}$ bond, but there is no experimental evidence in this current study to suggest such an involvement following excitation at 270 and $273 \mathrm{~nm}$.

Following excitation at $282 \mathrm{~nm}$, the inclusion of a $\tau_{3}=\infty$ and an approximately 0 fs component (Gaussian shown as the blue-dashed line) is necessary to accurately fit the data set. The channel associated with $\tau_{3}$ essentially resembles a step function. $\tau_{3}$ is highly consistent with the results reported by Longarte et al. ${ }^{59}$ following excitation at $283 \mathrm{~nm}$. However, these authors do not observe the 0 fs relaxation time that we associate with nonresonant signal/motion out of the Franck-Condon region nor do they see signs of such a process when pumping at $278 \mathrm{~nm}$. Instead, Longarte et al.'s TR-IY results at $278 \mathrm{~nm}$ contain an exponentially rising signal that they relate to an increase in the overall ionization cross-section.

We now return to the discussion regarding the ${ }^{1} \mathrm{~L}_{\mathrm{b}}$-state relaxation times. As mentioned earlier, the extracted time constant $\tau_{3}$ following excitation at $250 \mathrm{~nm}$ disagrees with Townsend $e t a l .{ }^{8}$ by nearly a factor of two. Likewise, $\tau_{3}$ differs quite significantly with the work of Longarte $e t a l .{ }^{59}$ following excitation at $260 \mathrm{~nm}$. Yet even with these disagreements, the nanosecond decay times associated with ${ }^{1} \mathrm{~L}_{\mathrm{b}}$-state activity following excitation at 270 and $273 \mathrm{~nm}$ broadly agree with both Townsend et al., who utilize a $273 \mathrm{~nm}$ pump, and Longarte et al., who employ pump wavelengths set at 269 and $272.5 \mathrm{~nm}$. However, a strict comparison to our results is not possible because neither Townsend et al. nor Longarte et al. report the ${ }^{1} \mathrm{~L}_{\mathrm{b}}$-state decay values at these wavelengths, just orders of magnitude or an infinite decay relative to the scanned region. One possible reason, as suggested earlier, for the discrepancies at 250 and $260 \mathrm{~nm}$ is the difference in observed pump-probe delay ranges. We simply note the observed trend among our obtained values to bolster these results. According to our study, the ${ }^{1} \mathrm{~L}_{\mathrm{b}}$ state becomes longer lived as the excitation energy decreases-transitioning from a $\tau_{3}=371 \pm 85$ ps lifetime at $241 \mathrm{~nm}$ to the nanosecond relaxation time observed at $273 \mathrm{~nm}$. This trend is also consistent with our previous work at $201 \mathrm{~nm}$, where an ${ }^{1} \mathrm{~L}_{\mathrm{b}}$-state lifetime of $\tau_{3}=23 \pm 5$ ps is reported. ${ }^{11}$

\section{Conclusions}

Three pump-probe spectroscopic experimental techniques were utilized to study the relaxation dynamics of indole in the gas phase at various excitation wavelengths. The first, TR-IY, 
revealed negligible excited-state fragmentation other than the $\mathrm{N}-\mathrm{H}$ dissociation for shorter excitation wavelengths. The second, TR-KER, afforded an analysis of the bimodal, steady-state H-atom kinetic energy spectra. High kinetic energy $\mathrm{H}$-atoms associated with relaxation along the ${ }^{1} \pi \sigma^{*}$ pathway began emanating from the $\mathrm{N}-\mathrm{H}$ moiety in the mid- to low-260 $\mathrm{nm}$ range, which is in strong agreement with the high resolution work of Ashfold et al. ${ }^{10}$ The lack of high kinetic energy $\mathrm{H}$-atoms at longer wavelengths than indicated implies insufficient energy to initiate $\mathrm{H}$-atom dissociation. However, for pump wavelengths centered at 241, 250, and $260 \mathrm{~nm}$, the ${ }^{1} \pi \sigma^{*}$ relaxation times exhibited a distinct trend of increasing from $515 \pm 63$ fs to $932 \pm 91 \mathrm{fs}$. This may be indicative of a potential energy barrier existing along the ${ }^{1} \pi \sigma^{*}$ relaxation pathway as predicted by ab initio calculations, ${ }^{8,19}$ but an alternative explanation is proposed below.

These results were complemented with a series of TR-PES studies at 241, 250, 260, 270, 273, and $282 \mathrm{~nm}$. The ${ }^{1} \mathrm{~L}_{\mathrm{a}}$-state contributions exhibited ultrafast decay times at the shorter wavelengths and time constants of a couple hundred femtoseconds at 270 and $273 \mathrm{~nm}$. No apparent contribution from the ${ }^{1} \mathrm{~L}_{\mathrm{a}}$ state is observed when exciting just above the ${ }^{1} \mathrm{~L}_{\mathrm{b}}$-state onset at $282 \mathrm{~nm}$. Time constants extracted from the ${ }^{1} \mathrm{~L}_{\mathrm{b}}$ signal contributions exhibited a dependence on pump wavelength: increasing from $371 \pm 85$ ps to the nanosecond range. In agreement with the results reported by Longarte et al., all ${ }^{1} \pi \sigma^{*}$-state relaxation times were $\sim 450$ fs. This result is intriguing in light of the systematic increase in lifetimes observed with TR-KER toward longer excitation wavelengths. We attribute this discrepancy to loss of the ionization window in the TR-PES measurement caused by the downward curving ${ }^{1} \pi \sigma^{*}$ potential energy curve and the upward trend of the ionic-state potential energy profiles. Moreover, this leads to the notion that motion in the vicinity of the ${ }^{1} \pi \sigma^{*} / \mathrm{S}_{0} \mathrm{CI}$, at extended $\mathrm{N}-\mathrm{H}$ distances, accounts for a significant portion of the relaxation dynamics.

As far as this experimental work is concerned, there is no evidence of ${ }^{1} \pi \sigma^{*}$-state involvement in the indole relaxation process following excitation at 270 and $273 \mathrm{~nm}$-in agreement with the ultrafast electron diffraction work of Zewail and co-workers. ${ }^{62}$

\section{Acknowledgements}

This work was supported by the University of Georgia Provosts Faculty Research Grant and the National Science Foundation grant\# NSF-CHE-1362237. The authors are also extremely grateful to Mr. D. R. Grimes and Mrs. Patricia Grimes for supporting this work through the Grimes Family Distinguished Graduate Fellowship Award, to the UGA Center for Undergraduate Research Opportunities (CURO) for supporting this work through the CURO Summer Fellowship program, and to Dr. Justin M. Turney for his help regarding theoretical aspects of the indole photophysics.

\section{Notes and references}

1 P. Meredith, B. J. Powell, J. Riesz, S. P. Nighswander-Rempel, M. R. Pederson and E. G. Moore, Soft Matter, 2006, 2, 37.
2 P. Meredith and T. Sarna, Pigm. Cell Res., 2006, 19, 572.

3 S. J. Harris, D. Murdock, Y. Zhang, T. A. A. Oliver, M. P. Grubb, A. J. Orr-Ewing, G. M. Greetham, I. P. Clark, M. Towrie, S. E. Bradforth and M. N. R. Ashfold, Phys. Chem. Chem. Phys., 2013, 15, 6567.

4 Y. Zhang, T. A. A. Oliver, M. N. R. Ashfold and S. E. Bradforth, Faraday Discuss., 2012, 157, 141.

5 D. Murdock, S. J. Harris, T. N. V. Karsili, G. M. Greetham, I. P. Clark, M. Towrie, A. J. Orr-Ewing and M. N. R. Ashfold, J. Phys. Chem. Lett., 2012, 3, 3715.

6 T. A. A. Oliver, Y. Zhang, M. N. R. Ashfold and S. E. Bradforth, Faraday Discuss., 2011, 150, 439.

7 F. F. Crim, Faraday Discuss., 2012, 157, 9.

8 R. Livingstone, O. Schalk, A. E. Boguslavskiy, G. R. Wu, L. T. Bergendahl, A. Stolow, M. J. Paterson and D. Townsend, J. Chem. Phys., 2011, 135, 194307.

9 A. Iqbal and V. G. Stavros, J. Phys. Chem. A, 2010, 114, 68.

10 M. G. D. Nix, A. L. Devine, B. Cronin and M. N. R. Ashfold, Phys. Chem. Chem. Phys., 2006, 8, 2610.

11 T. J. Godfrey, H. Yu and S. Ullrich, J. Chem. Phys., 2014, 141, 044314.

12 H. Lami, J. Chem. Phys., 1977, 67, 3274.

13 H. Lami, Chem. Phys. Lett., 1977, 48, 447.

14 N. A. Borisevich and T. F. Raichuyonok, Dokl. Phys., 2007, $52,405$.

15 J. R. Platt, J. Chem. Phys., 1949, 17, 484.

16 M. N. R. Ashfold, G. A. King, D. Murdock, M. G. D. Nix, T. A. A. Oliver and A. G. Sage, Phys. Chem. Chem. Phys., 2010, 12, 1218.

17 A. L. Sobolewski and W. G. Domcke, J. Phys. Chem. A, 2007, 111, 11725.

18 M. F. Lin, C. M. Tseng, Y. T. Lee and C. K. Ni, J. Chem. Phys., 2005, 123, 124303.

19 A. L. Sobolewski and W. Domcke, Chem. Phys. Lett., 1999, 315, 293.

20 G. A. Bickel, D. R. Demmer, E. A. Outhouse and S. C. Wallace, J. Chem. Phys., 1989, 91, 6013.

21 G. Berden, W. L. Meerts and E. Jalviste, J. Chem. Phys., 1995, 103, 9596.

22 J. Kupper, D. W. Pratt, W. L. Meerts, C. Brand, J. Tatchen and M. Schmitt, Phys. Chem. Chem. Phys., 2010, 12, 4980.

23 T. L. O. Barstis, L. I. Grace, T. M. Dunn and D. M. Lubman, J. Phys. Chem., 1993, 97, 5820.

24 P. R. Callis, J. T. Vivian and L. S. Slater, Chem. Phys. Lett., 1995, 244, 53.

25 J. Hager and S. C. Wallace, J. Phys. Chem., 1983, 87, 2121.

26 D. M. Sammeth, S. X. Yan, L. H. Spangler and P. R. Callis, J. Phys. Chem., 1990, 94, 7340.

27 R. Bersohn, U. Even and J. Jortner, J. Chem. Phys., 1984, 80, 1050.

28 J. R. Cable, J. Chem. Phys., 1990, 92, 1627.

29 J. W. Hager, D. R. Demmer and S. C. Wallace, J. Phys. Chem., 1987, 91, 1375.

30 J. M. Hollas, Spectrochim. Acta, 1963, 19, 753.

31 Y. Nibu, H. Abe, N. Mikami and M. Ito, J. Phys. Chem., 1983, 87, 3898.

32 B. J. Fender, D. M. Sammeth and P. R. Callis, Chem. Phys. Lett., 1995, 239, 31. 
33 V. A. Povedailo and D. L. Yakovlev, J. Appl. Spectrosc., 2008, 75, 336.

34 C. Brand, J. Kupper, D. W. Pratt, W. L. Meerts, D. Krugler, J. Tatchen and M. Schmitt, Phys. Chem. Chem. Phys., 2010, 12, 4968.

35 A. Giussani, M. Merchan, D. Roca-Sanjuan and R. Lindh, J. Chem. Theory Comput., 2011, 7, 4088.

36 H. Lippert, H. H. Ritze, I. V. Hertel and W. Radloff, Chem. Phys. Lett., 2004, 398, 526.

37 N. L. Evans, H. Yu, G. M. Roberts, V. G. Stavros and S. Ullrich, Phys. Chem. Chem. Phys., 2012, 14, 10401.

38 N. L. Evans and S. Ullrich, J. Phys. Chem. A, 2010, 114, 11225. 39 P. Kruit and F. H. Read, J. Phys. E: Sci. Instrum., 1983, 16, 313. 40 W. C. Wiley and I. H. McLaren, Rev. Sci. Instrum., 1955, 26, 1150. 41 D. M. P. Holland, M. A. MacDonald, M. A. Hayes, P. Baltzer, B. Wannberg, M. Lundqvist, L. Karlsson and W. von Niessen, J. Phys. B: At., Mol. Opt. Phys., 1996, 29, 3091.

42 R. Locht, K. Hottmann, G. Hagenow, W. Denzer and H. Baumgartel, Chem. Phys. Lett., 1992, 190, 124.

43 L. Serrano-Andrés and B. O. Roos, J. Am. Chem. Soc., 1996, 118, 185.

44 A. C. Borin and L. Serrano-Andrés, Chem. Phys., 2000, 262, 253. 45 J. Catalan, P. Perez and A. U. Acuna, J. Mol. Struct., 1986, 142, 179. 46 L. N. Domelsmith, L. L. Munchausen and K. N. Houk, J. Am. Chem. Soc., 1977, 99, 4311.

47 J. H. D. Eland, Int. J. Mass Spectrom. Ion Phys., 1969, 2, 471.
48 L. J. Dolby, G. Hanson and T. Koenig, J. Org. Chem., 1976, 41, 3537.

49 T. A. A. Oliver, G. A. King and M. N. R. Ashfold, Phys. Chem. Chem. Phys., 2011, 13, 14646.

50 B. Valeur and G. Weber, Photochem. Photobiol., 1977, 25, 441.

51 Y. Yamamoto and J. Tanaka, Bull. Chem. Soc. Jpn., 1972, 45, 1362.

52 M. R. Eftink, L. A. Selvidge, P. R. Callis and A. A. Rehms, J. Phys. Chem., 1990, 94, 3469.

53 R. L. Rich, Y. Chen, D. Neven, M. Négrerie, F. Gai and J. W. Petrich, J. Phys. Chem., 1993, 97, 1781.

54 E. Jalviste and N. Ohta, J. Chem. Phys., 2004, 121, 4730.

55 B. C. Dian, A. Longarte and T. S. Zwier, J. Chem. Phys., 2003, 118, 2696.

56 P. R. Callis, J. Chem. Phys., 1991, 95, 4230.

57 R. Montero, A. P. Conde, A. Longarte and F. Castaño, ChemPhysChem, 2010, 11, 3420.

58 V. Ovejas, M. Fernández-Fernández, R. Montero, F. Castaño and A. Longarte, J. Phys. Chem. Lett., 2013, 4, 1928.

59 R. Montero, A. P. Conde, V. Ovejas, F. Castano and A. Longarte, J. Phys. Chem. A, 2012, 116, 2698.

60 J. M. Turney, personal communication.

61 M. Barbatti and S. Ullrich, Phys. Chem. Chem. Phys., 2011, 13, 15492.

62 S. T. Park, A. Gahlmann, Y. He, J. S. Feenstra and A. H. Zewail, Angew. Chem., Int. Ed., 2008, 47, 9496. 